\title{
VARIACIÓN MORFOLÓGICA Y ANATÓMICA EN POBLACIONES MEXICANAS DE PSEUDOTSUGA (PINACEAE)
}

\author{
Valentín J. Reyes Hernández ${ }^{1}$, J. Jesús Vargas Hernández ${ }^{1}$, \\ Javier López Upton ${ }^{1}$ y Humberto Vaquera Huerta ${ }^{2}$ \\ ${ }^{1}$ Programa Forestal, Colegio de Postgraduados \\ km 36.5 Carretera México-Texcoco, 56230 Montecillo, México \\ ${ }^{2}$ Programa en Estadística, Colegio de Postgraduados \\ km. 36.5 Carretera México-Texcoco, 56230 Montecillo, México \\ vreyes@colpos.mx
}

\section{RESUMEN}

Con base en características morfológicas y anatómicas de conos, hojas y ramillas se evaluó el patrón geográfico de variación en poblaciones naturales del género Pseudotsuga en México. Las muestras de órganos vegetativos y reproductivos se colectaron en un total de 293 árboles procedentes de 19 localidades de las diferentes regiones geográficas en las cuales se distribuye el género en nuestro país. Se analizaron los caracteres considerados como importantes desde el punto de vista de la sistemática, de tal forma que fueran útiles para tratar de esclarecer los límites de los taxa de este género en México. El análisis de varianza mostró que existe una fuerte diferenciación entre las poblaciones en la mayoría de las variables consideradas, con 22 a $92 \%$ de la varianza total a este nivel. La región geográfica de procedencia tuvo un papel importante en la separación de las muestras, lo que implica la existencia de ecotipos diferentes de Pseudotsuga en el territorio de la República. Debido a que la variabilidad atribuible a los taxa putativos fue menor que la correlacionada con las regiones geográficas, se concluye que no existen bases morfológicas suficientes para separar las especies de Pseudotsuga propuestas para México con anterioridad.

Palabras clave: componentes de varianza, diferenciación morfológica, ecotipos, México, Pinaceae, Pseudotsuga, taxa putativos.

\begin{abstract}
Using morphological and anatomical traits of cones, leaves and branchlets, the geographic pattern of variation was evaluated in natural populations of Pseudotsuga in Mexico. Vegetative and reproductive samples were collected from a total of 293 trees in 19
\end{abstract}


populations located throughout the native range of this genus in Mexico. Characters considered as important from a taxonomic point of view were included in order to verify the taxonomic boundaries of the components of this genus in the region. A strong differentiation among populations was detected for most morphological and anatomical traits, with 22 to $92 \%$ of the phenotypic variation residing at this level. The geographical region of origin had an important role in the separation of samples, suggesting the presence of geographic ecotypes of Pseudotsuga in Mexico. Given the fact that differentiation associated with putative taxa was much lower than differentiation associated to geographic regions, it is concluded that there are no morphological bases to separate the Pseudotsuga species that have been proposed earlier for Mexico.

Key words: ecotypes, Mexico, morphological differentiation, Pinaceae, Pseudotsuga, putative taxa, variance components.

\section{INTRODUCCIÓN}

Pseudotsuga Carr. es uno de los géneros de árboles de mayor importancia económica a nivel mundial y desde mediados del Pleistoceno ha sido el principal componente de los bosques del oeste de Norteamérica (Hermann y Lavender, 1990). Sus especies se localizan exclusivamente en dos grandes regiones del hemisferio norte, en el oeste de Norteamérica y en el sureste de Asia (Farjon, 1990). De acuerdo con Little (1974), el área natural actual de Pseudotsuga en el continente americano alcanza $55^{\circ}$ de latitud norte en Columbia Británica en Canadá, llegando a los $19^{\circ} \mathrm{N}$ en el centro-sur de México, pero recientemente Debreczy y Racz (1995) descubrieron una nueva localidad en la Sierra de Juárez, en el norte de Oaxaca a $17^{\circ} 10^{\prime} \mathrm{N}$, la cual representa el extremo sur en la distribución conocida del género.

Pseudotsuga forma bosques puros y continuos de gran extensión en la región costera y en las cordilleras del interior de Canadá y Estados Unidos, en cambio en el suroeste de este último país y en México su área se vuelve discontinua y fragmentada, formando poblaciones aisladas, la mayoría de las veces en convivencia con otras especies arbóreas (Farjon, 1990). Debido a la diversidad topográfica y microambiental, al aislamiento de las localidades y a su posición geográfica marginal con respecto al área más contínua de distribución ubicada más al norte del continente, es lógico suponer que el género Pseudotsuga pudiera haber estado sujeto en México a procesos de diferenciación genética. En los trabajos descriptivos de Flous (1934) y de Martínez (1963) se propone y se acepta la existencia de cuatro especies de Pseudotsuga en el territorio de la República: P. macrolepis Flous, P. rehderi Flous, $P$. flahaulti Flous y $P$. guinieri Flous. Sin embargo, con base en la escasa divergencia morfológica y en la falta de separación geográfica de las áreas de distribución de las especies propuestas, algunos taxónomos consideran que todo el 
complejo mexicano forma parte de P. menziesii var. glauca (Little, 1979; Hermann, 1982; Farjon, 1990), taxon cuya área principal de distribución se extiende a las Montañas Rocallosas de Estados Unidos y Canadá. Las posibles variantes morfológicas en las plantas mexicanas se atribuyen a la variación clinal y ecotípica a nivel de las poblaciones (Hermann, 1982; Farjon, 1990). A pesar de esta controversia, hasta la fecha no existe información detallada sobre la magnitud y estructura de la variabilidad en características morfológicas de Pseudotsuga en México.

Considerando el aislamiento y la fragmentación en su área de distribución, y tomando como base la descripción de Martínez (1963), la Norma Oficial Mexicana NOM-ECOL-059 considera al género Pseudotsuga como un grupo taxonómico sujeto a protección especial desde el año de 1994 (Anónimo, 1994), categoría que fue ratificada en el año 2002 (Anónimo, 2002). No obstante la protección legal establecida en la NOM, muchas de las poblaciones naturales de este género están sometidas a fuertes presiones antropogénicas, especialmente aquéllas situadas en el límite de la frontera agrícola o en zonas de aprovechamiento forestal (maderero o de otra índole), por lo que existe un riesgo elevado de destrucción y pérdida de recursos genéticos valiosos.

La situación anterior hace evidente la necesidad de obtener información básica acerca del patrón geográfico de la variación morfológica y anatómica en las poblaciones de Pseudotsuga en México, con el fin de encontrar límites taxonómicos claros y proponer alternativas de conservación. Actualmente existen técnicas que permiten definir relaciones filogenéticas y categorías taxonómicas con base en marcadores genéticos moleculares (Moritz y Hillis, 1996; Martínez, 1997); los trabajos de Furman et al. (1997), Gernandt y Liston (1999) y Liston et al. (1999) son ejemplos de ello. Sin embargo, existen fuertes controversias sobre el uso indiscriminado de estas técnicas en la sistemática, ya que requieren también del apoyo de los métodos tradicionales de identificación y clasificación de organismos con base en caracteres morfológicos (Martínez, 1997; Hillis, 1987). No tiene mucho sentido establecer una separación taxonómica basada en marcadores moleculares si las diferencias entre individuos no son evidentes y distinguibles en el campo a partir de su morfología.

Por lo anterior, el presente estudio se llevó a cabo con los objetivos de: 1) determinar la magnitud relativa de la variación existente en 16 características morfológicas y anatómicas entre y dentro de 19 poblaciones mexicanas del género Pseudotsuga; 2) evaluar la posible existencia de un patrón geográfico de diferenciación en estos caracteres; y 3) discutir las implicaciones ecológicas y taxonómicas de la estructura de la variabilidad observada en las poblaciones de Pseudotsuga. 


\section{MATERIALES Y MÉTODOS}

Revisión de ejemplares de herbario y obtención de muestras

Para la realización de este trabajo se llevó a cabo una revisión de ejemplares en el herbario de la Universidad Autónoma de Chapingo (CHAP), con la finalidad de ubicar, de acuerdo con la clasificación de Martínez (1963), algunas de las localidades donde se registran los morfotipos de las especies aceptadas por dicho autor para el género Pseudotsuga en México. A partir de esta información se muestreó un total de 19 poblaciones naturales de los diferentes taxa putativos reconocidos por dicho autor a lo largo del área de distribución natural del género en el país (Fig. 1), incluyendo La Sierra Madre Occidental (Región I), la Sierra Madre Oriental (Región II) y el centro del país (Región III). En el Cuadro 1 se presentan los datos de localización precisa de los sitios de colecta.

Se definió una estructura jerárquica del método, tomando en cuenta los niveles de regiones geográficas, localidades dentro de las regiones, árboles dentro de

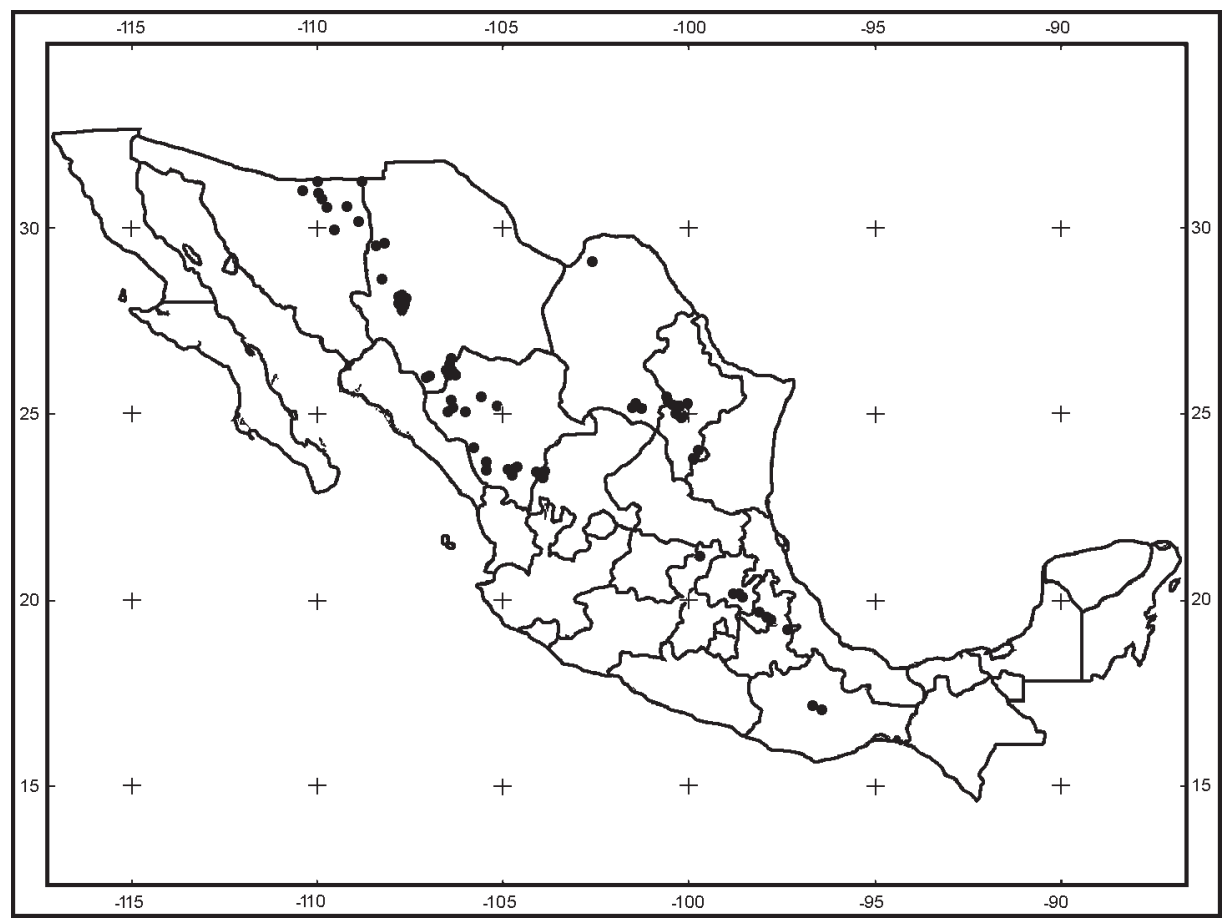

Fig. 1. Distribución natural conocida del género Pseudotsuga en México. (Mapa proporcionado por el M. C. Alberto Domínguez). 
Cuadro 1. Localidades mexicanas de Pseudotsuga incluidas en el estudio de variación morfológica y anatómica. Para los taxa putativos se sigue la la clasificación de Martínez (1963). Reg. = Región geográfica.

\begin{tabular}{|c|c|c|c|c|c|}
\hline \multirow{2}{*}{ Reg. } & \multirow{2}{*}{ Localidad } & \multirow{2}{*}{ Taxon putativo } & \multicolumn{2}{|c|}{ Localización } & \multirow{2}{*}{$\begin{array}{l}\text { Elevación } \\
(\mathrm{m} \text { s.n.m.) }\end{array}$} \\
\hline & & & Lat. N & Long. O & \\
\hline \multirow[t]{7}{*}{ I } & Río Chico, Madera, Chih. & P. rehderi & $29^{\circ} 37^{\prime} 03^{\prime \prime}$ & $108^{\circ} 09^{\prime} 42^{\prime \prime}$ & 1900 \\
\hline & Cerro La Candelaria, Madera, Chih. & P. flahaulti & $29^{\circ} 30^{\prime} 40^{\prime \prime}$ & $108^{\circ} 25^{\prime} 45^{\prime \prime}$ & 2500 \\
\hline & Bajío Largo, Guerrero, Chih. & P. guinieri & $28^{\circ} 09^{\prime} 41^{\prime \prime}$ & $107^{\circ} 44^{\prime} 16^{\prime \prime}$ & 2650 \\
\hline & Río Verde, Balleza, Chih. & P. rehderi & $26^{\circ} 16^{\prime} 48^{\prime \prime}$ & $106^{\circ} 27^{\prime} 22^{\prime \prime}$ & 2450 \\
\hline & Cerro Mohinora, Gpe. y Calvo, Chih. & P. macrolepis & $25^{\circ} 57^{\prime} 41^{\prime \prime}$ & $107^{\circ} 02^{\prime} 14^{\prime \prime}$ & 3050 \\
\hline & $\begin{array}{l}\text { Sierra La Candela, Sta. María del } \\
\text { Oro, Dgo. }\end{array}$ & P. macrolepis & $25^{\circ} 27^{\prime} 51^{\prime \prime}$ & $105^{\circ} 33^{\prime} 42^{\prime \prime}$ & 2900 \\
\hline & $\begin{array}{l}\text { Mesa de los Leones, Huachichiles, } \\
\text { Dgo. }\end{array}$ & P. guinieri & $24^{\circ} 05^{\prime} 40^{\prime \prime}$ & $105^{\circ} 44^{\prime} 50^{\prime \prime}$ & 3000 \\
\hline \multirow[t]{7}{*}{ II } & Sta. Anita, Arteaga, Coah. & P. flahaulti & $25^{\circ} 27^{\prime} 02^{\prime \prime}$ & $100^{\circ} 34^{\prime} 11^{\prime \prime}$ & 2400 \\
\hline & San Antonio de las Alazanas, Coah. & P. flahaulti & $25^{\circ} 20^{\prime} 27^{\prime \prime}$ & $100^{\circ} 33^{\prime} 57^{\prime \prime}$ & 2600 \\
\hline & Sierra La Marta, Rayones, N.L. & P. rehderi & $25^{\circ} 12^{\prime} 26^{\prime \prime}$ & $100^{\circ} 22^{\prime} 27^{\prime \prime}$ & 3000 \\
\hline & Ejido La Providencia, Saltillo, Coah. & P. flahaulti & $25^{\circ} 09^{\prime} 36^{\prime \prime}$ & $101^{\circ} 11^{\prime} 57^{\prime \prime}$ & 2750 \\
\hline & $\begin{array}{l}\text { San Francisco del Javier, } \\
\text { Galeana, N.L. }\end{array}$ & P. macrolepis & $24^{\circ} 58^{\prime} 04^{\prime \prime}$ & $100^{\circ} 20^{\prime} 09^{\prime \prime}$ & 2800 \\
\hline & La Lagunilla, Galeana, N.L. & P. macrolepis & $24^{\circ} 56^{\prime} 42^{\prime \prime}$ & $100^{\circ} 16^{\prime} 02^{\prime \prime}$ & 2600 \\
\hline & Ejido 18 de Marzo, Galeana, N.L. & P. macrolepis & $24^{\circ} 52^{\prime} 57^{\prime \prime}$ & $100^{\circ} 11^{\prime} 34^{\prime \prime}$ & 2190 \\
\hline \multirow[t]{5}{*}{ III } & Rancho El Pardo, Tlaxco, Tlax. & P. macrolepis & $19^{\circ} 38^{\prime} 55^{\prime \prime}$ & $98^{\circ} 03^{\prime} 11^{\prime \prime}$ & 2960 \\
\hline & Barranca La Rosa, Terrenate, Tlax. & P. macrolepis & $19^{\circ} 31^{\prime} 42^{\prime \prime}$ & $97^{\circ} 54^{\prime} 58^{\prime \prime}$ & 2700 \\
\hline & La Caldera, Ixtacamaxtitlán, Pue. & P. macrolepis & $19^{\circ} 30^{\prime} 23^{\prime \prime}$ & $97^{\circ} 52^{\prime} 10^{\prime \prime}$ & 2950 \\
\hline & Axopilco, Altzayanca, Tlax. & P. macrolepis & $19^{\circ} 27^{\prime} 42^{\prime \prime}$ & $97^{\circ} 46^{\prime} 18^{\prime \prime}$ & 2760 \\
\hline & $\begin{array}{l}\text { Apizaquito, San José Cuauhtémoc, } \\
\text { Pue. }\end{array}$ & P. macrolepis & $19^{\circ} 12^{\prime} 10^{\prime \prime}$ & $97^{\circ} 18^{\prime} 41^{\prime \prime}$ & 3350 \\
\hline
\end{tabular}


localidades y muestras dentro de árboles. En cada localidad se obtuvieron muestras de ramas, hojas y estróbilos de entre 12 y 17 árboles a lo largo de gradientes altitudinales, de exposición y de pendiente del terreno; se seleccionaron individuos sanos y separados al menos 50 metros uno del otro para reducir las relaciones de parentesco entre los mismos. Los especímenes se tomaron de ramas expuestas en la parte media de la copa, se etiquetaron en forma individual y se transportaron en bolsas de papel para su análisis posterior. Se registraron además las coordenadas geográficas con un geoposicionador y la elevación promedio del sitio con un altímetro (Cuadro 1). El muestreo se realizó en diferentes fechas en los años 2000 y 2001, debido a la separación geográfica de las localidades y a la imposibilidad de encontrar órganos reproductivos en todas ellas en el mismo año.

Evaluación de variables morfológicas y anatómicas

En el estudio se incluyeron las variables morfológicas y anatómicas de conos, hojas y ramillas consideradas como importantes desde el punto de vista taxonómico de acuerdo con las descripciones de Flous (1934) y Martínez (1963), con el propósito de verificar si es posible agrupar de manera efectiva a las poblaciones en los cuatro taxa correspondientes.

En una muestra de cinco conos cerrados (antes de que abran las escamas) por árbol se registró la longitud y el diámetro basal, realizándose ambas mediciones con un vernier digital con aproximación de $0.1 \mathrm{~mm}$; posteriormente, los estróbilos fueron secados a una temperatura de $40-50^{\circ} \mathrm{C}$ durante $12-24$ horas para permitir la separación de las escamas. De la parte central de cada uno de ellos se tomó una muestra de 3-4 escamas con su bráctea íntegra, para medir su longitud y ancho así como la longitud total de la bráctea, la longitud de la espina central y la de las espinas laterales (Fig. 2). Con estos datos se obtuvieron los valores promedio por cono para cada característica, y con ellos se calcularon varios índices de forma de las estructuras del cono, incluyendo el índice de forma del cono (proporción entre el largo y el diámetro de cono) y de la escama (proporción entre el largo y el ancho de la escama), la relación bráctea-escama (proporción entre la longitud de la bráctea y la longitud de la escama) y la relación espina central-espina lateral (proporción entre la longitud de la espina central y la longitud de la espina lateral de las brácteas).

Los rasgos morfológicos y anatómicos de hojas y ramillas se evaluaron en una muestra de cinco especímenes del último año de crecimiento por árbol. La longitud total de las hojas se midió con una regla graduada en milímetros, y el número de hileras de estomas de cuantificó en la sección izquierda del envés en la parte media de la hoja con la ayuda de un microscopio estereoscópico con aumento 10x. Posteriormente, las hojas y ramillas fueron sometidas a un proceso de rehidratación, 

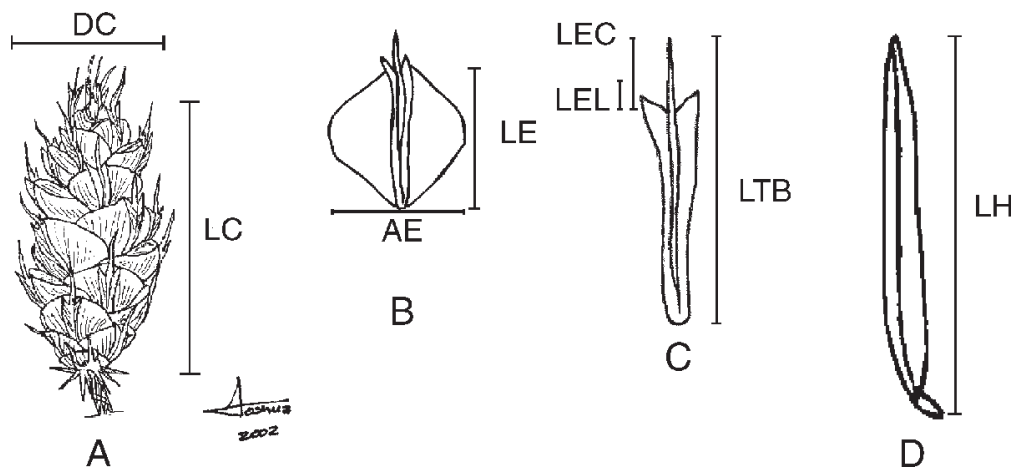

Fig. 2. Variables morfológicas medidas en: A. Cono; B. Escama; C. Bráctea; D. Hoja. DC = diámetro de cono; $\mathrm{LC}=$ longitud de cono; $\mathrm{AE}$ = ancho de escama; $\mathrm{LE}=$ longitud de escama; LEC = longitud de la espina central; LTB = longitud total de la bráctea; LEL = longitud de la espina lateral; $\mathrm{LH}=$ longitud de hoja.

mediante inmersión en agua con jabón común durante un período de 10-12 horas a una temperatura de $50^{\circ} \mathrm{C}$ dentro de una estufa. Una vez rehidratadas, se efectuaron cortes transversales en la parte media de la hoja y a una distancia aproximada de $2 \mathrm{~cm}$ del ápice en las ramillas; después se colocaron en un portaobjetos con una gota de ácido láctico y se calentaron en la flama de un mechero de alcohol para facilitar la visibilidad de las estructuras de interés. En estas preparaciones temporales se determinó la estructura de la subepidermis en las hojas (continua o discontinua), así como el número de canales resiníferos y la forma de la sección transversal (circular o pentagonal) en las ramillas, con ayuda de un microscopio óptico con aumentos 10x y 40x (Fig. 3).

Análisis estadístico

Debido a que las características anatómicas de la estructura de la subepidermis y del número de hileras estomatales en las hojas, así como la forma de la sección transversal y el número de canales resiníferos en las ramillas, son variables binarias $\mathrm{y}$ discretas, respectivamente, se hicieron transformaciones de las mismas. El número de canales resiníferos y de hileras estomatales se expresó a manera de la raíz cuadrada del valor original. Sin embargo, los resultados del análisis de varianza con las variables así tratadas fueron similares a los obtenidos con los datos originales, por lo que se decidió presentar estos últimos. Las variables de tipo binario (continuidad de la subepidermis y forma de la sección transversal) fueron convertidas a proporciones por árbol y, posteriormente, modificadas con la función arco seno (Cochran, 1940; Kung, 1988). Nuevamente los resultados del análisis de varianza 


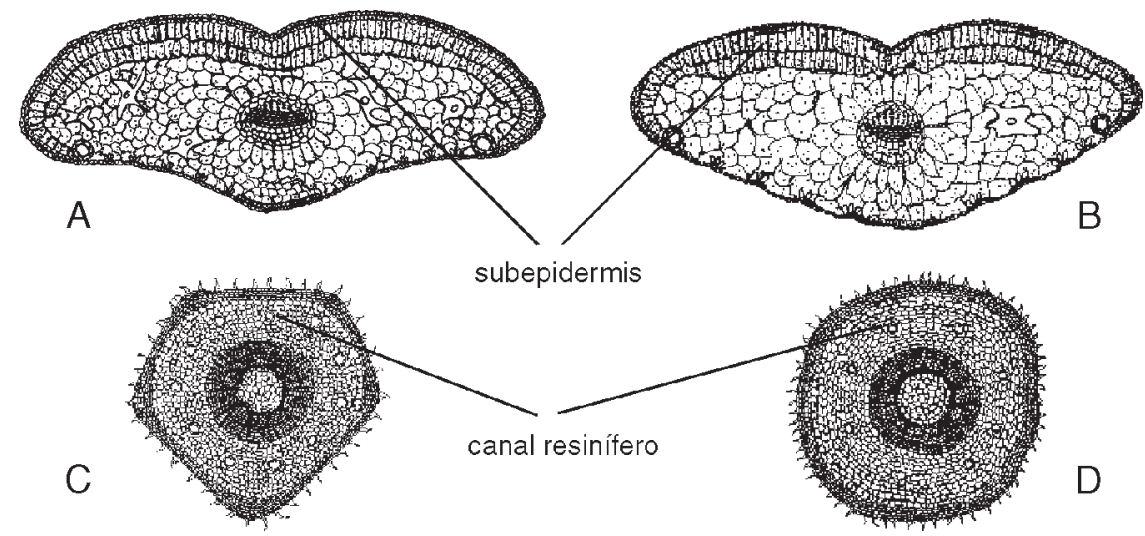

Fig. 3. Variables anatómicas evaluadas en hojas y ramillas: A. Hoja con subepidermis continua; B. Hoja con subepidermis discontinua; C. Ramilla en sección transversal pentagonal; D. Ramilla en sección transversal circular.

fueron similares para los datos originales (en proporciones) y para los transformados, por lo que sólo se presentan los primeros.

El análisis de varianza se llevó a cabo considerando el modelo 2 de efectos aleatorios con muestras de clasificación jerárquica o muestreo anidado (Snedecor y Cochran, 1981). Inicialmente se consideró un modelo simplificado que incluyó únicamente a las poblaciones y a los árboles dentro de ellas, con el propósito de determinar sus respectivos componentes de varianza. El modelo utilizado en este caso fue el siguiente:

$$
\mathrm{Y}_{\mathrm{ijk}}=\mu+\mathrm{L}_{\mathrm{i}}+\mathrm{A}_{\mathrm{j}(\mathrm{i})}+\varepsilon_{\mathrm{k}(\mathrm{ij})}
$$

Donde:

$\mathrm{Y}_{\mathrm{ijk}}=$ Valor observado de la característica en la k-ésima muestra del j-ésimo árbol en la i-ésima localidad;

$\mu=$ Valor promedio de la característica;

$\mathrm{L}_{\mathrm{i}} \quad=$ Efecto aleatorio de la i-ésima población $\left[\mathrm{E}\left(\mathrm{L}_{\mathrm{i}}\right)=0, \operatorname{Var}\left(\mathrm{L}_{\mathrm{i}}\right)=\sigma_{\mathrm{L}}^{2}\right]$;

$\mathrm{A}_{\mathrm{j}(\mathrm{i})}=$ Efecto aleatorio del $\mathrm{j}$-ésimo árbol dentro de la i-ésima población $\left[E\left(A_{j(i)}\right)=0, \operatorname{Var}\left(A_{j(i)}\right)=\sigma_{A j(i)}^{2}\right]$;

$\varepsilon_{\mathrm{k}(\mathrm{ij})}=$ Error aleatorio de muestreo dentro de árboles $\left[\mathrm{E}\left(\varepsilon_{\mathrm{k}(\mathrm{ij})}\right)=0\right.$, $\left.\operatorname{Var}\left(\varepsilon_{\mathrm{k}(\mathrm{ij})}\right)=\sigma_{\varepsilon}^{2}\right]$. 
En las variables convertidas a proporciones por árbol el modelo sólo incluyó el nivel de poblaciones, ya que en este caso la varianza del error representa la varianza entre árboles dentro de poblaciones y no existe error de muestreo dentro de árboles (las muestras originales fueron utilizadas para estimar la proporción por árbol). Posteriormente, con el propósito de comparar la importancia relativa de las regiones geográficas versus el grupo taxonómico putativo en la distribución de la varianza existente, se utilizaron dos modelos de análisis alternativos. En el primero (modelo $\mathrm{A}_{1}$ ) se incluyó a las regiones y a las poblaciones contenidas en ellas además de los otros componentes del modelo original, de la siguiente forma:

$$
\mathrm{Y}_{\mathrm{ijkl}}=\mu+\mathrm{R}_{\mathrm{i}}+\mathrm{L}_{\mathrm{j}(\mathrm{i})}+\mathrm{A}_{\mathrm{k}(\mathrm{ij})}+\varepsilon_{\mathrm{ijk} l}
$$

Donde:

$$
\begin{aligned}
& \mathrm{Y}_{\mathrm{ijk} 1}=\text { Valor observado de la característica en la 1-ésima muestra del } \\
& \text { k-ésimo árbol de la j-ésima población en la i-ésima región } \\
& \text { geográfica; } \\
& \mu \quad=\text { Valor promedio de la característica; } \\
& \mathrm{R}_{\mathrm{i}}=\text { Efecto aleatorio de la i-ésima región geográfica } \\
& {\left[\mathrm{E}\left(\mathrm{R}_{\mathrm{i}}\right)=0 \text {, Var }\left(\mathrm{R}_{\mathrm{i}}\right)\right]=\sigma_{\mathrm{R}}^{2} \text {; }} \\
& \mathrm{L}_{\mathrm{j}(\mathrm{i})}=\text { efecto aleatorio de la j-ésima población dentro de la i-ésima región } \\
& {\left[\mathrm{E}\left(\mathrm{L}_{\mathrm{j}(\mathrm{i})}\right)=0, \operatorname{Var}\left(\mathrm{L}_{\mathrm{j}(\mathrm{i})}\right)=\sigma_{\mathrm{L}(\mathrm{R})}^{2}\right]} \\
& \mathrm{A}_{\mathrm{k}(\mathrm{ij})}=\text { Efecto aleatorio del k-ésimo árbol de la j-ésima población en la } \\
& \text { i-ésima región geográfica } \\
& {\left[\mathrm{E}\left(\mathrm{A}_{\mathrm{k}(\mathrm{ij})}\right)=0, \operatorname{Var}\left(\mathrm{A}_{\mathrm{k}(\mathrm{ij})}\right)=\sigma_{\mathrm{A}(\mathrm{LR})}^{2}\right]} \\
& \varepsilon_{\mathrm{ijkl}}=\begin{array}{l}
\text { Error aleatorio de muestreo dentro de árboles (definido } \\
\text { anteriormente). }
\end{array}
\end{aligned}
$$

En el segundo caso (modelo $\mathrm{A}_{2}$ ) se incluyó a los taxa putativos en lugar de las regiones geográficas, utilizando la misma estructura del modelo $\mathrm{A}_{1}$, pero sustituyendo los efectos aleatorios de regiones y de localidades dentro de regiones geográficas, por los de taxa $\left[\mathrm{E}\left(\mathrm{T}_{\mathrm{i}}\right)=0, \operatorname{Var}\left(\mathrm{T}_{\mathrm{i}}\right)=\sigma_{\mathrm{Ti}_{\mathrm{i}}}^{2}\right]$ y de localidades dentro de taxa.

En una primera etapa del análisis se utilizó el procedimiento GLM del SAS ${ }^{\circledR}$ (Anónimo, 1998) para determinar la significancia estadística de cada uno de los efectos incluidos en los modelos. Posteriormente se usó el procedimiento VARCOMP con la opción de Máxima Verosimilitud Restringida (REML) para obtener los componentes de varianza estimados con cada uno de los modelos considerados en el estudio. Además, se calcularon las correlaciones entre los valores promedio por población de las características morfológicas y anatómicas con los datos geográficos (altitud, latitud y longitud) de los sitios de colecta, con la finalidad 
de determinar la probable asociación de las variables evaluadas con los gradientes geográficos existentes, producto de la distribución discontinua del género.

\section{RESULTADOS}

Variación entre y dentro de poblaciones

Los resultados del análisis efectuado con el modelo simplificado mostraron que existe una variación significativa a nivel poblacional en todas las características morfológicas y anatómicas consideradas ( $p \leq 0.05)$, concentrándose a ese nivel entre 22 y $92 \%$ de la varianza fenotípica total (Cuadro 2); además, con excepción de la longitud de la hoja, la aportación relativa de las poblaciones a la varianza total en todas las demás variables fue igual o mayor que la aportación de los árboles dentro de las localidades (Cuadro 2). Lo anterior indica que en los rodales de Pseudotsuga analizados existe una fuerte diferenciación en las características morfológicas y anatómicas de conos, hojas y ramillas. Aun en el caso de la longitud de las hojas se muestra esta tendencia, con $35 \%$ de la variación total a este nivel, a pesar de que también existe una amplia variabilidad entre árboles dentro de los sitios (casi 55\%).

Un aspecto importante a destacar es que, con excepción de la relación entre el tamaño de las espinas en las brácteas, la varianza a nivel de árboles fue relativamente baja en todos los caracteres considerados. Esto contrasta con los resultados obtenidos en la mayoría de los estudios realizados en otras especies de coníferas, principalmente pinos, en las cuales dicho componente representa el mayor aporte (López et al., 1993; Romero, 1995; Santiago, 1995). Sin embargo, Prieto (1992) encontró que en el género Picea en México, la mayor variación en siete de diez indicadores morfológicos evaluados se presentó entre poblaciones, resultados que coinciden con los obtenidos en este estudio. Al igual que Pseudotsuga, Picea también tiene una distribución geográfica discontinua y fragmentada en México.

Las características morfológicas que presentaron mayor varianza relativa entre localidades fueron la longitud de la espina central, la longitud de la bráctea y el diámetro de cono, con valores cercanos o mayores de 50\% (Cuadro 2). Entre las variables anatómicas con una situación similar se encuentran la forma de la sección transversal y el número de canales resiníferos en las ramillas, así como la continuidad de la subepidermis y el número de las hileras estomatales en las hojas, con una aportación superior a 50\% en ese nivel (Cuadro 2). Lo anterior implica, por un lado, que las poblaciones de Pseudotsuga incluidas en el presente estudio han estado expuestas a un proceso de diferenciación morfológica, posiblemente como resultado de la separación geográfica derivada de su distribución relictual. Como sugiere McDonald (1993), uno de los efectos del aislamiento entre las áreas parciales 
Cuadro 2. Componentes de varianza estimados entre y dentro de poblaciones para las diferentes características morfológicas y anatómicas de conos, hojas y ramillas de Pseudotsuga.

\begin{tabular}{|c|c|c|c|c|}
\hline \multirow[b]{2}{*}{ Característica } & \multicolumn{3}{|c|}{ Componente de varianza $(\%)$} & \multirow[b]{2}{*}{$\begin{array}{c}\text { Varianza } \\
\text { total }\end{array}$} \\
\hline & Poblaciones* & $\begin{array}{c}\text { Árboles } \\
\text { dentro de } \\
\text { poblaciones }\end{array}$ & $\begin{array}{c}\text { Muestras } \\
\text { dentro de } \\
\text { árboles }\end{array}$ & \\
\hline Diámetro de cono & 48.68 & 28.39 & 22.93 & 5.5451 \\
\hline Longitud de cono & 43.59 & 33.54 & 22.87 & 101.3537 \\
\hline Índice de forma del cono & 35.90 & 35.90 & 28.20 & 0.1289 \\
\hline Longitud de escama & 43.21 & 36.04 & 20.75 & 7.7439 \\
\hline Ancho de escama & 39.01 & 33.22 & 27.77 & 5.3015 \\
\hline Longitud de la bráctea & 51.05 & 31.69 & 17.26 & 15.2745 \\
\hline Longitud de espina lateral & 41.57 & 18.00 & 40.43 & 0.8555 \\
\hline Longitud de espina central & 68.40 & 21.50 & 10.10 & 3.5613 \\
\hline Índice de forma de la escama & 35.03 & 32.66 & 32.31 & 0.0074 \\
\hline Relación bráctea-escama & 43.52 & 28.62 & 27.86 & 0.0143 \\
\hline $\begin{array}{l}\text { Relación espina central-espina } \\
\text { lateral }\end{array}$ & 22.51 & 13.23 & 64.26 & 1.5618 \\
\hline Longitud de hoja & 35.18 & 54.86 & 9.96 & 10.1498 \\
\hline Hileras de estomas & 70.17 & 13.55 & 16.28 & 2.0634 \\
\hline Canales resiníferos & 67.90 & 16.12 & 15.98 & 2.6477 \\
\hline Subepidermis foliar ${ }^{\dagger}$ & 59.53 & 40.46 & - & 0.1984 \\
\hline Forma de la sección transversal ${ }^{\dagger}$ & 93.57 & 6.43 & - & 0.2560 \\
\hline
\end{tabular}

* Significativo con $p \leq 0.05 .{ }^{\dagger}$ En estas características se utilizaron proporciones por árbol, por lo que no existen muestras dentro de árboles (ver texto). 
de la misma especie es el aumento de la diversificación genética entre ellas y el desarrollo de endemismos, lo que se presenta en algunas asociaciones vegetales subalpinas de la Sierra Madre Oriental en el noreste de México, donde es común encontrar a Pseudotsuga.

Comparación de modelos (regiones geográficas vs. taxa putativos)

Los resultados obtenidos al comparar los dos modelos alternativos mostraron la existencia de una varianza significativa $(p \leq 0.05)$ a nivel de las regiones geográficas en siete de las 16 características evaluadas. En cambio, al considerar a los taxa putativos sólo cinco de éstas fueron estadísticamente significativas. Además, en los caracteres con aportación significativa las regiones representan entre 16 y $66 \%$ de la variación fenotípica total, mientras que los taxa sólo contribuyen entre 15 y $28 \%$ (Cuadro 3). Los rasgos que presentaron diferencias significativas a nivel de regiones fueron la longitud de la espina lateral y de la espina central, la relación bráctea-escama, la longitud de la hoja, el número de hileras estomatales y de canales resiníferos, y la forma de la sección transversal de las hojas. Cuatro de estos atributos coinciden con el grupo de caracteres que mostró mayor variación entre las poblaciones en el modelo original. Las regiones geográficas de origen de las muestras aportan al menos el doble de varianza relativa al total (Modelo $\mathrm{A}_{1}$ ), en comparación con la aportación relativa de los taxa en el modelo alternativo (Modelo $\mathrm{A}_{2}$ ), excepto la "relación bráctea-escama", la cual presenta valores muy similares en ambos modelos (Cuadro 3). Lo anterior indica que en este grupo de caracteres el proceso de diferenciación entre las poblaciones está más asociado a su distribución geográfica que a la posición taxonómica asignada originalmente por Flous (1934) y Martínez (1963).

El último autor (op. cit.) reconoce que Pseudotsuga es un complejo taxonómico difícil de separar debido a que sus atributos morfológicos externos son muy variables y señala que algunos indicadores de la estructura interna de hojas y ramillas, como la continuidad de la subepidermis, la forma de la sección transversal y el número de canales resiníferos, pueden ser más útiles para agrupar taxonómicamente a los componentes de este género. Los resultados de nuestro estudio confirman algunas de las observaciones realizadas por este autor. Por ejemplo, en las características morfológicas de conos y hojas se encontró una amplia variación, cuyo elevado porcentaje se observa entre poblaciones. Además, salvo el caso de la longitud de las espinas lateral y central en las brácteas, esta variabilidad no tuvo relación alguna con las regiones geográficas o los taxa putativos. En cambio, con excepción de la continuidad en la subepidermis foliar, los atributos anatómicos internos mostraron una mayor correlación geográfica. Sin embargo, contrario a lo expuesto por Martínez (1963), no parece existir una congruencia elevada entre estos caracteres y los taxa propuestos. La varianza relativa que aporta la región geográfica 
Cuadro 3. Comparación de los modelos alternativos (regiones vs. taxa putativos) de distribución de la variación (componentes de varianza) entre poblaciones para diferentes características morfológicas y anatómicas de conos, hojas y ramillas de Pseudotsuga.

\begin{tabular}{|c|c|c|c|c|}
\hline \multirow{3}{*}{ Variable } & \multicolumn{4}{|c|}{ Componente de varianza $(\%)$} \\
\hline & \multicolumn{2}{|c|}{ Modelo $\mathrm{A}_{1}$} & \multicolumn{2}{|c|}{ Modelo $\mathrm{A}_{2}$} \\
\hline & Regiones & $\begin{array}{c}\text { Poblaciones } \\
\text { dentro de } \\
\text { regiones }\end{array}$ & Taxa & $\begin{array}{c}\text { Poblaciones } \\
\text { dentro de } \\
\text { taxa }\end{array}$ \\
\hline Diámetro de cono & $1.59^{\mathrm{NS}}$ & 47.33 & $0^{\mathrm{NS}}$ & 48.68 \\
\hline Longitud de cono & $4.27^{\mathrm{NS}}$ & 39.98 & $0^{\mathrm{NS}}$ & 43.59 \\
\hline Índice de forma del cono & $0^{\mathrm{NS}}$ & 35.91 & $0^{\mathrm{NS}}$ & 35.91 \\
\hline Longitud de escama & $0^{\mathrm{NS}}$ & 43.20 & $0^{\mathrm{NS}}$ & 43.20 \\
\hline Ancho de escama & $0^{\mathrm{NS}}$ & 39.01 & $0^{\mathrm{NS}}$ & 39.01 \\
\hline Longitud de la bráctea & $0^{\mathrm{NS}}$ & 51.05 & $4.43^{\mathrm{NS}}$ & 47.21 \\
\hline Longitud de espina lateral & $25.52 *$ & 21.52 & $17.15^{*}$ & 25.42 \\
\hline Longitud de espina central & $47.53^{*}$ & 25.72 & $26.66^{*}$ & 43.08 \\
\hline Índice de forma de la escama & $4.77^{\mathrm{NS}}$ & 31.10 & $2.89^{\mathrm{NS}}$ & 32.63 \\
\hline Relación bráctea-escama & $16.52^{*}$ & 29.94 & $17.04 *$ & 28.27 \\
\hline $\begin{array}{l}\text { Relación espina central- } \\
\text { espina lateral }\end{array}$ & $4.13^{\mathrm{NS}}$ & 19.46 & $3.93^{\mathrm{NS}}$ & 19.60 \\
\hline Longitud de hoja & $30.18^{*}$ & 11.52 & $15.75^{*}$ & 22.44 \\
\hline Hileras de estomas & $65.28^{*}$ & 12.12 & $27.94 *$ & 43.57 \\
\hline Canales resiníferos & $39.58^{*}$ & 31.45 & $0^{\mathrm{NS}}$ & 67.90 \\
\hline Subepidermis foliar & $16.49^{\mathrm{NS}}$ & 45.43 & $0^{\mathrm{NS}}$ & 59.53 \\
\hline Forma de la sección transversal & 66.87 & 28.17 & $22.96^{\mathrm{NS}}$ & 70.89 \\
\hline
\end{tabular}

* Significativo con $\alpha=0.05$. NS No significativo con $\alpha=0.05$. Nota: La suma de los componentes de varianza estimados difiere ligeramente de los valores presentados entre poblaciones en el Cuadro 2, debido al procedimiento iterativo que utiliza el método de Máxima Verosimilitud Restringida al estimarlos. 
de la cual proceden las muestras es mayor que la atribuible al efecto del taxon putativo. Por lo tanto, aunque existe una amplia separación en los caracteres morfológicos y anatómicos entre las poblaciones de Pseudotsuga incluidas en este estudio, dicha variación fenotípica está asociada en mayor grado con el sitio de colecta que con el taxon asignado por Martínez (1963).

Estructura geográfica de la variación en las características morfológicas y anatómicas

Ocho de las 15 características evaluadas presentaron una correlación significativa $(p \leq 0.05)$ con al menos una de las variables geográficas de los sitios de colecta (Cuadro 4). Siete de ellas se asociaron significativamente con la latitud, y cinco también con la longitud; en cambio, sólo una lo hizo con la altitud. En todos los casos, las correlaciones con la latitud y longitud fueron aproximadamente de la misma magnitud y signo, lo cual indica que ambas representan un gradiente ambiental similar. Destacan la longitud de la espina lateral, de la espina central y de la hoja, así como la relación bráctea-escama, las cuales mostraron valores de " $r$ " entre 0.55 y 0.83 con la latitud y la longitud del sitio (Cuadro 4); solamente el diámetro del cono presentó una vinculación significativa con la altitud del sitio de colecta.

Las cifras de correlación obtenidas indican que el tamaño promedio de la hoja aumenta gradualmente hacia el norte y el oeste del área de distribución natural de Pseudotsuga en México (Fig. 4). Como se observa en el Cuadro 5, las poblaciones ubicadas hacia el norte del país (regiones I y II) registran valores más altos en la longitud foliar en comparación con aquellas ubicadas en la zona centro (región III). Por otro lado, el número de hileras estomatales tiende a disminuir conforme aumenta la latitud. En las localidades del norte de México (regiones I y II) los árboles presentan en promedio un menor número de líneas de estomas a pesar de tener una mayor longitud de la hoja que las del centro (Cuadro 5). La longitud de la espina central y lateral también tiende a aumentar hacia el norte y el oeste del área muestreada, pero se reduce conforme aumenta la altitud del sitio de colecta.

Debido a las condiciones del estudio, es difícil establecer los factores ambientales que están influyendo en la estructura geográfica de la varianza observada en estas características. Sin embargo, la temperatura, la precipitación pluvial y la humedad atmosférica son las variables climáticas que generalmente se han asociado con la latitud, la altitud y la longitud en la región geográfica muestreada (Rzedowski, 1978), por lo que se infiere que uno o varios de estos factores ambientales podrían estar asociados con el patrón geográfico detectado en los caracteres morfológicos del género Pseudotsuga en México. 
Cuadro 4. Coeficientes de correlación de Pearson (r) estimados entre las características morfológicas y anatómicas de conos, hojas y ramillas de Pseudotsuga y las variables geográficas del sitio de colecta $(\mathrm{n}=19)$.

\begin{tabular}{|c|c|c|c|}
\hline \multirow{2}{*}{ Característica } & \multicolumn{3}{|c|}{ Variable geográfica } \\
\hline & Altitud & Latitud & Longitud \\
\hline Diámetro de cono & $0.54^{*}$ & -0.19 & -0.32 \\
\hline Longitud de cono & 0.44 & -0.09 & -0.28 \\
\hline Longitud de escama & 0.44 & 0.01 & -0.13 \\
\hline Ancho de escama & 0.29 & 0.20 & 0.11 \\
\hline Longitud de la bráctea & 0.20 & 0.37 & 0.29 \\
\hline Longitud de espina lateral & -0.37 & $0.75^{*}$ & $0.65^{*}$ \\
\hline Longitud de espina central & -0.34 & $0.81 *$ & $0.81 *$ \\
\hline Índice de forma de la escama & 0.33 & -0.27 & -0.40 \\
\hline Relación bráctea-escama & -0.24 & $0.55^{*}$ & $0.62 *$ \\
\hline $\begin{array}{l}\text { Relación espina central- } \\
\text { espina lateral }\end{array}$ & 0.24 & -0.26 & -0.09 \\
\hline Longitud de hoja & -0.21 & $0.79 *$ & $0.83 *$ \\
\hline Hileras de estomas & 0.42 & $-0.74 *$ & -0.38 \\
\hline Canales resiníferos & 0.38 & $-0.48^{*}$ & $-0.68 *$ \\
\hline Subepidermis foliar & -0.25 & -0.32 & -0.37 \\
\hline Forma de la sección transversal & -0.44 & $0.53 *$ & 0.14 \\
\hline
\end{tabular}

* Valores absolutos de $\mathrm{r}$ mayores o iguales a 0.45 son significativos con $\mathrm{p}=0.05$. 
Acta Botanica Mexicana 70: 47-67 (2005)
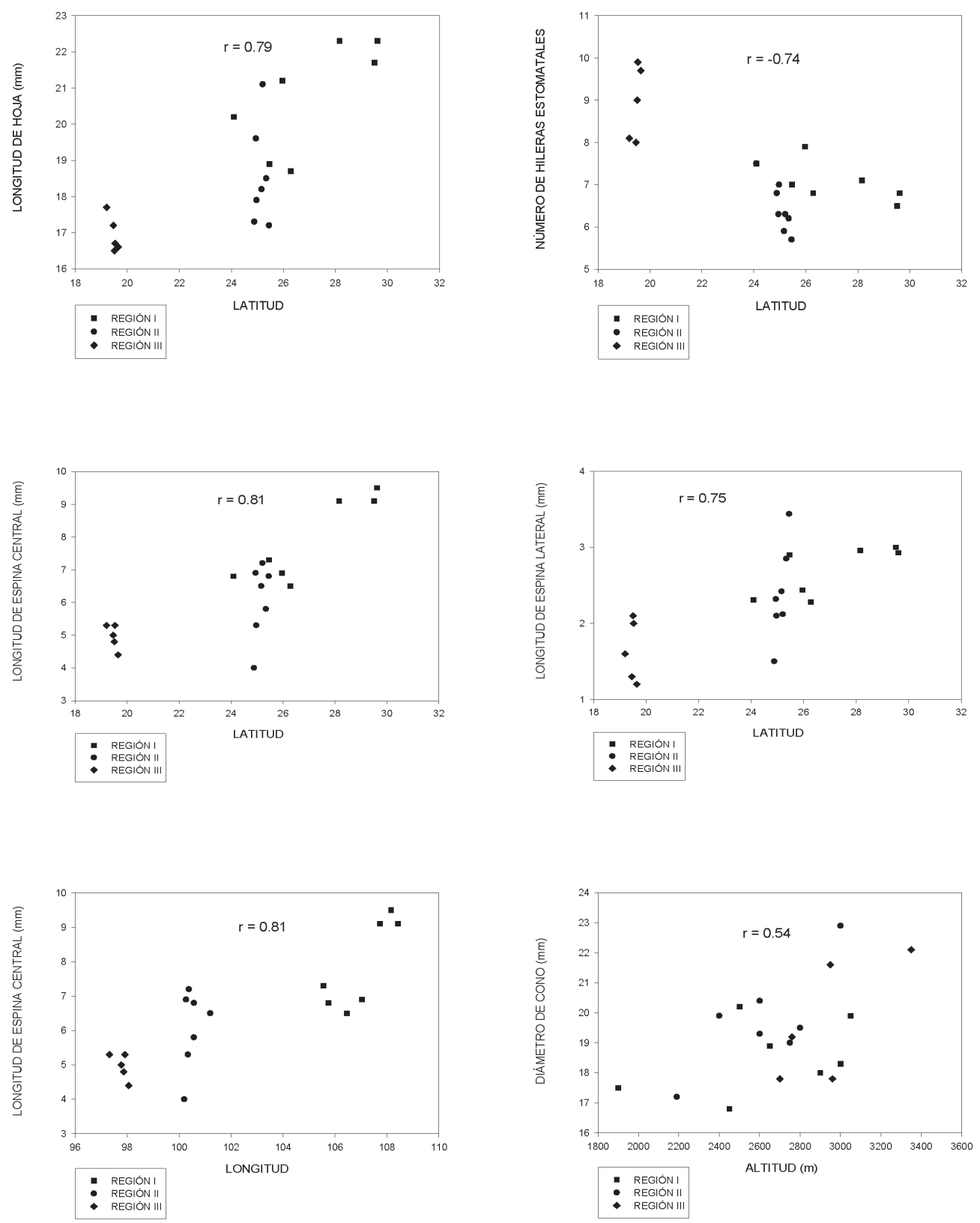

Fig. 4. Correlaciones lineales estadísticamente significativas $(\alpha=0.05)$ entre las variables morfológicas y anatómicas de conos, hojas y ramillas de Pseudotsuga y las variables geográficas de los sitios de colecta. 
Cuadro 5. Valores promedio $(\overrightarrow{\mathrm{x}})$ y extremos por región geográfica de las características morfológicas y anatómicas de conos, hojas y ramillas en poblaciones mexicanas de Pseudotsuga.

\begin{tabular}{|c|c|c|c|c|c|c|}
\hline \multirow{2}{*}{ Característica } & \multicolumn{2}{|c|}{ REGIÓN I } & \multicolumn{2}{|c|}{ REGIÓN II } & \multicolumn{2}{|c|}{ REGIÓN III } \\
\hline & $\overline{\mathrm{x}}$ & Extremos & $\overline{\mathrm{x}}$ & Extremos & $\overline{\mathrm{x}}$ & Extremos \\
\hline Diámetro de cono (mm) & 18.5 & $16.8-20.2$ & 19.7 & $17.1-22.9$ & 19.7 & $17.8-22.1$ \\
\hline Longitud de cono (mm) & 50.0 & $40.2-53.5$ & 56.3 & $40.0-68.8$ & 53.0 & $48.9-57.7$ \\
\hline Índice de forma del cono & 2.7 & $2.4-2.9$ & 2.8 & $2.3-3.1$ & 2.7 & $2.5-3.0$ \\
\hline Longitud de escama (mm) & 21.9 & $18.7-23.8$ & 23.0 & $18.5-25.3$ & 22.3 & $21.2-24.7$ \\
\hline Ancho de escama (mm) & 21.1 & $18.5-23.6$ & 21.1 & $17.6-23.2$ & 20.8 & $19.6-22.3$ \\
\hline Longitud de la bráctea (mm) & 28.5 & $23.4-31.4$ & 28.1 & $20.9-32.4$ & 26.4 & $24.3-28.7$ \\
\hline Longitud de espina lateral $(\mathrm{mm})^{*}$ & 2.7 & $2.3-3.0$ & 2.4 & $1.52-3.42$ & 1.6 & $1.2-2.1$ \\
\hline Longitud de espina central $(\mathrm{mm}) *$ & 7.9 & $6.5-9.5$ & 6.1 & $4.04-7.21$ & 4.9 & $4.4-5.3$ \\
\hline Índice de forma de la escama & 1.0 & $1.0-1.1$ & 1.1 & $1.0-1.2$ & 1.1 & $1.0-1.1$ \\
\hline Relación bráctea-escama* & 1.3 & $1.3-1.4$ & 1.2 & $1.09-1.34$ & 1.2 & $1.1-1.2$ \\
\hline $\begin{array}{l}\text { Relación espina central- } \\
\text { espina lateral }\end{array}$ & 3.2 & $2.6-3.5$ & 2.8 & $2.1-3.7$ & 3.5 & $2.5-4.4$ \\
\hline Longitud de hoja $(\mathrm{mm})^{*}$ & 20.8 & $18.7-22.3$ & 18.5 & $17.2-21.1$ & 16.9 & $16.5-17.7$ \\
\hline Hileras de estomas (núm.)* & 7.1 & $6.5-7.9$ & 6.3 & $5.7-7.0$ & 8.9 & $8.0-9.9$ \\
\hline Canales resiníferos (núm.)* & 10.4 & $8.9-12.4$ & 12.5 & $11.1-13.4$ & 12.1 & $11.2-12.7$ \\
\hline Subepidermis foliar ${ }^{\dagger}$ & 0.54 & $0-1.0$ & 0.65 & $0.32-1.0$ & 0.99 & $0.97-1.0$ \\
\hline Forma de la sección transversal* & 0.49 & $0-1.0$ & 1.00 & $1.0-1.0$ & 0.02 & $0-0.06$ \\
\hline
\end{tabular}

*Significativas con $\alpha=0.05$ con base en los resultados del análisis de varianza del modelo $A_{1}$. †Proporción de árboles en la población con subepidermis foliar discontinua. 'Proporción de árboles en la población con ramillas de sección transversal pentagonal. 


\section{IMPLICACIONES ECOLÓGICAS Y TAXONÓMICAS}

En síntesis, los resultados del estudio muestran que existe una elevada diferenciación morfológica entre las poblaciones mexicanas de Pseudotsuga, asociada fundamentalmente con las discontinuidades geográficas de su área de distribución natural, y que parece estar ocasionada por el aislamiento y separación de las localidades, incluso dentro de cada región. La divergencia en algunas características como la longitud de la hoja y el número de hileras estomatales podría considerarse como una respuesta adaptativa a las condiciones ambientales del sitio, en virtud de su correlación significativa con la latitud del lugar de origen.

En estudios de variación genética llevados a cabo en Pseudotsuga menziesii (Mirb.) Franco en los Estados Unidos de América, la mayor parte de la varianza existente entre localidades ha sido relacionada con variables ecológicas, geográficas y fisiográficas (Rehfeldt, 1983). Por ejemplo, en el norte de Idaho, Rehfeldt (1979) detectó grandes diferencias entre poblaciones separadas únicamente por $140 \mathrm{~m}$ de altitud, $1^{\circ}$ de latitud, $1.5^{\circ}$ de longitud. Al parecer, dichas poblaciones muestran adaptaciones fisiológicas a sus ambientes particulares (Rehfeldt, 1983), situación que ha propiciado su diferenciación. Los altos niveles de variabilidad encontrados entre las localidades incluidas en nuestro estudio sugieren que, dado que éstas crecen en ambientes diferentes, han tenido que adaptarse a factores ecológicos contrastantes, lo cual se refleja en sus atributos morfológicos y anatómicos. Esta divergencia fenotípica fue la que en un momento propició que Flous (1934) y Martínez (1963) determinaran y aceptaran la existencia de varias especies de Pseudotsuga en el continente americano, especialmente en México.

Sin embargo, de acuerdo con el concepto morfológico de especie (Cain, 1954, citado por Crisci, 1994), las poblaciones de Pseudotsuga incluidas en el presente estudio no pueden ser consideradas como entidades diferentes de este nivel, debido a que no reúnen características morfológicas lo suficientemente contrastantes para ser distinguibles. Los rasgos evaluados en el presente estudio, que fueron considerados importantes por Flous (1934) y Martínez (1963) para separar a las especies, registran traslape en los valores promedios de las poblaciones tanto a nivel de los taxa putativos como de las regiones geográficas consideradas, por lo que no existe una separación precisa entre tales especies. A pesar de que en algunos atributos anatómicos es más notoria la separación entre poblaciones, la variación que éstos presentan es atribuible en mayor grado al lugar de colecta de las muestras que al taxon putativo (Cuadro 3).

Lo anterior permite concluir que no existen bases morfológicas y anatómicas suficientes para establecer una separación clara y precisa de los taxa propuestos por Flous (1934) y Martínez (1963). En función de la distribución geográfica discontinua del género dentro y entre las regiones analizadas en este trabajo, las 
poblaciones locales pueden postularse como ecotipos diferentes con patrón de variación clinal dentro de cada región; sin embargo, para corroborar este punto es necesario establecer pruebas o ensayos genéticos con germoplasma de estas procedencias en un ambiente común uniforme.

Debido al alto nivel de diferenciación morfológica y anatómica entre las poblaciones de Pseudotsuga analizadas, es necesario establecer estrategias precisas de conservación (in situ y ex situ) para ellas dentro de cada región geográfica. El nivel de variabilidad local y el posible valor adaptativo de algunas de estas características permite considerar a cada población como un recurso genético de gran valor potencial. La necesidad de protección es particularmente urgente en la región del centro de México, ya que es aquí donde los árboles de Pseudotsuga están expuestos a mayores presiones que amenazan su permanencia. Además, las poblaciones de esta zona son las que muestran una mayor diferenciación con respecto al resto de los sitios muestreados (Fig. 4 y Cuadro 5).

\section{AGRADECIMIENTOS}

Este trabajo forma parte del proyecto de investigación "Diversidad genética y conservación de Pseudotsuga en México", realizado con apoyo económico del CONACYT a través del proyecto clave 33617-B. Se agradece al M. C. Carlos Ramírez Herrera, al Ing. Celestino Flores López e Ing. Manuel Mapula Larreta por su ayuda en el trabajo de campo; al Ing. David Josué Meza Juárez por su apoyo en la obtención de las muestras en laboratorio y realización de los esquemas; así como a todas las personas que de alguna forma participaron durante el desarrollo del presente trabajo.

\section{LITERATURA CITADA}

Anónimo. 1994. Norma Oficial Mexicana NOM-059-ECOL-1994 que determina las especies $\mathrm{y}$ subespecies de flora y fauna silvestres terrestres y acuáticas en peligro de extinción y que establece especificaciones para su protección. Diario Oficial de la Federación. Mayo de 1994. 54 pp.

Anónimo. 1998. SAS/STAT Guide for personal computers. Version 8.0. SAS Institute Inc Cary, NC.

Anónimo. 2002. Segunda sección-SEMARNAT. Norma Oficial Mexicana NOM-059-ECOL2001. Protección ambiental-especies nativas de México de flora y fauna silvestrescategorías de riesgo y especificaciones para su inclusión, exclusión o cambio - lista de especies en riesgo. Diario Oficial de la Federación. Marzo de 2002. 85 pp. 
Cochran, W. G. 1940. The analysis of variance when experimental errors follow the Poisson or binomial laws. Ann. Math. Stat. 11: 335-347.

Crisci, J. 1994. La especie: realidad y conceptos. In: Llorente J. e I. Luna (eds.). Taxonomía biológica. Ediciones Científicas Universitarias. Fondo de Cultura Económica. México, D.F. pp. 53-64.

Debreczy, Z. e I. Racz. 1995. New species and varieties of conifers from Mexico. Phytologia 78: 217-243.

Farjon, A. 1990. Pinaceae: drawings and descriptions of the genera Abies, Cedrus, Pseudolarix, Keteleeria, Nothotsuga, Tsuga, Cathaya, Pseudotsuga, Larix and Picea. Koeltz Scientific Books. Königstein. pp. 177-191.

Flous, F. 1934. Diagnoses d'espèces et variétés nouvelles de Pseudotsuga americains. Travaux du Laboratoire Forestier de Toulouse. Tomo I, Vol. 2, Art. VI. Toulouse. 18 pp.

Furman, B. J., D. Grattapaglia, W. S. Dvorak y D. M. O'Malley. 1997. Analysis of genetic relationship of Central American and Mexican pines using RAPD markers that distinguish species. Molecular Ecology 6: 321-331.

Gernandt, D. S y A. Liston. 1999. Internal transcribed spacer region evolution in Larix and Pseudotsuga (Pinaceae). Amer. J. Bot. 86(5): 711-723.

Hermann, R. K. 1982. The genus Pseudotsuga: historical records and nomenclature. Special Publication 2a. School of Forestry. Forest Research Laboratory. Oregon State University. Corvallis. 29 pp.

Hermann, R. K. y D. P. Lavender. 1990. Pseudotsuga menziesii (Mirb.) Franco. In: Burns R. y B. H. Honkala (coord. téc.). Silvics of North America. Vol. 1, Conifers. USDA Forest Service. Washington, D.C. pp. 527-540.

Hillis, D. M. 1987. Molecular versus morphological approaches to systematics. Ann. Rev. Ecol. Syst. 18: 23-42.

Kung, F. H. 1988. Application of data transformation in forest genetics. Silvae Gen. 37(2): 45-49.

Little, E. L. Jr. 1979. Checklist of United States trees (native and naturalized). USDA Forest Service. Agricultural Handbook N 541. 375 pp.

Liston, A., W. A. Robinson, D. Piñero, y E. R. Álvarez-Buylla. 1999. Phylogenetics of Pinus (Pinaceae) based on nuclear ribosomal DNA internal transcribed spacer region sequences. Mol. Phyl. Evol. 11: 95-109.

López U., J., J. Jasso M., J. Vargas H. y J. C. Ayala S. 1993. Variación de características morfológicas en conos y semillas de Pinus greggii Engelm. Agrociencia 3(1): 81-95.

Martínez, M. 1963. Las pináceas mexicanas. Universidad Nacional Autónoma de México. $3 \mathrm{a}$ ed. México, D.F. pp. 27-74.

Martínez, M. 1997. Sistemática molecular: comparación entre diferentes métodos y sus aplicaciones. Bol. Soc. Bot. Méx. 60: 123-136.

McDonald, J. A. 1993. Phytogeography and history of the alpine-subalpine flora of northeastern México. In: Ramamoorthy T. P., R. Bye, A. Lot y J. Fa (eds.). Biological diversity of Mexico: origins and distribution. Oxford Univ. Press. Nueva York. pp. 681-703. 
Moritz, C. y D. M. Hillis. 1996. Molecular systematics: context and controversies. In: Hillis, D. M., C. Moritz y B. K. Mable (eds.). Molecular systematics. 2a ed. Sinauer Associates Inc. Massachussets. pp: 1-12.

Prieto M., E. 1992. Variación morfológica en conos y semillas de 13 poblaciones naturales de Picea (Pinaceae) en México. Tesis Profesional. División de Ciencias Forestales. Universidad Autónoma Chapingo. Chapingo, México. 73 pp.

Rehfeldt, G. E. 1979. Ecological adaptations in Douglas-fir (Pseudotsuga menziesii var. glauca) populations. I. North Idaho and northeast Washington. Heredity 43(3): 383397.

Rehfeldt, G. E. 1983. Genetic variability within Douglas-fir populations: implications for tree improvement. Silvae Gen. 32(1-2): 9-14.

Romero M., R. 1995. Variación morfológica en conos y semillas en poblaciones naturales de Pinus durangensis Mtnz. Tesis profesional. División de Ciencias Forestales. Universidad Autónoma Chapingo. Chapingo, México. 94 pp.

Rzedowski, J. 1978. Vegetación de México. Ed. Limusa. México, D.F. 431 pp.

Santiago C., M. 1995. Variación de caracteres morfológicos y anatómicos de acículas en poblaciones naturales de Pinus durangensis Mtnz. Tesis profesional. División de Ciencias Forestales. Universidad Autónoma Chapingo. Chapingo, México. 88 pp.

Snedecor, W. G. y G. W. Cochran. 1981. Métodos estadísticos. Ed. Continental. México, D.F. $703 \mathrm{pp}$. 